Supporting Information

\title{
Emission from Charge Recombination during the Pulse Radiolysis of Arylethynylpyrenes
}

Shingo Samori, Sachiko Tojo, Mamoru Fujitsuka, Shu-Wen Yang, ${ }^{\dagger}$ Tong-Ing Ho, ${ }^{\dagger}$ Jye-Shane Yang, ${ }^{\dagger}$, and Tetsuro Majima*

The Institute of Scientific and Industrial Research (SANKEN), Osaka University

Mihogaoka 8-1, Ibaraki, Osaka 567-0047, Japan

${ }^{\dagger}$ Department of Chemistry, National Taiwan University, Taipei, Taiwan 10617

E-mail: majima@sanken.osaka-u.ac.jp

\section{Contents}

1. Figure S1. Transient absorption spectra observed during the laser flash photolysis of REPy $(0.2 \mathrm{mM})$ in Ar-saturated Bz. 


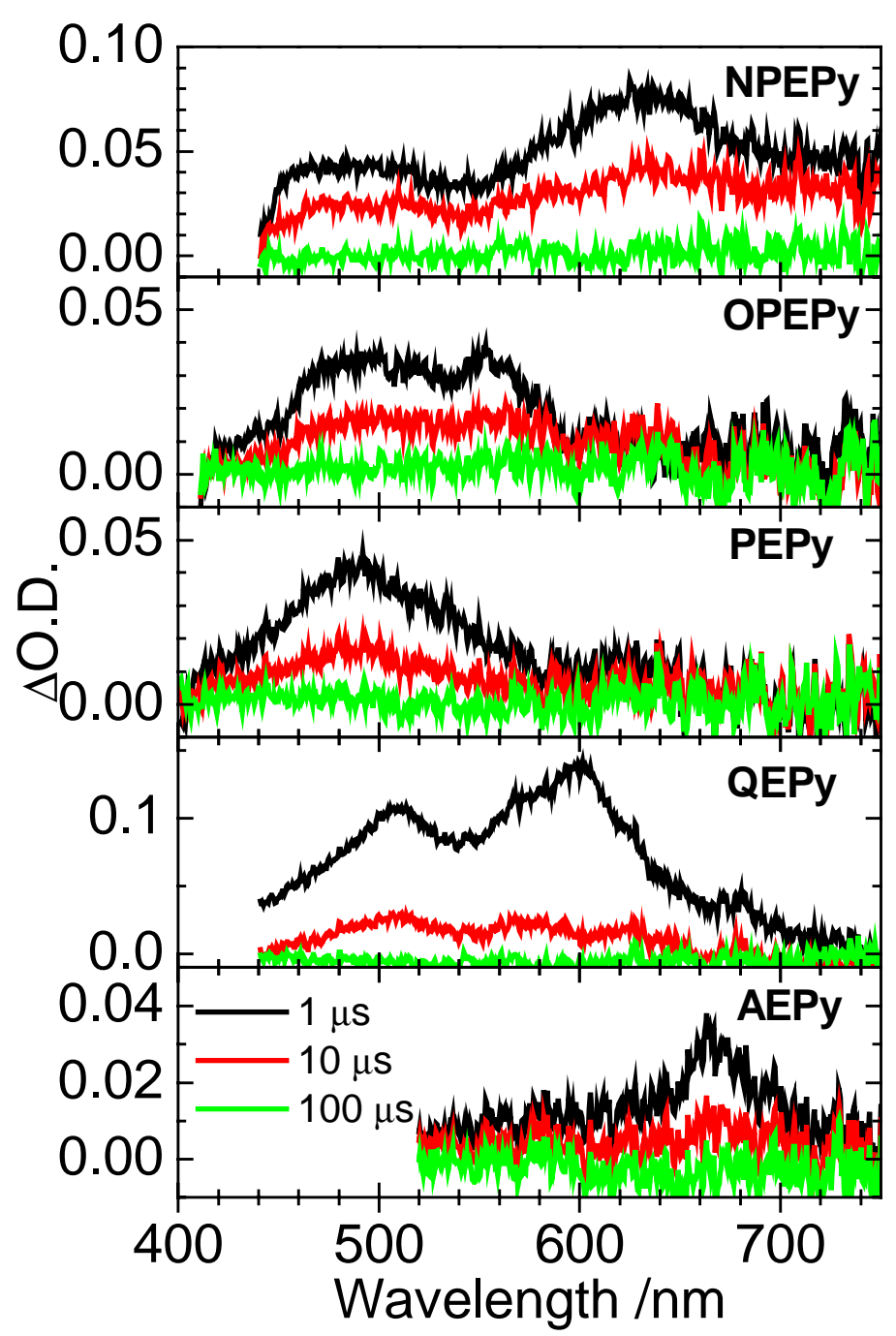

Figure S1. Transient absorption spectra observed at time $t=1$ (black line), 10 (red line), and 100 (green line) $\mu$ s after a laser flash (5 ns) during the laser flash photolysis of REPy $(0.2 \mathrm{mM})$ in Ar-saturated Bz. 